\title{
Characterizing the Atmospheres of Hot Jupiters: From Spectra to Multi-Color Maps
}

\author{
Heather A. Knutson \\ Harvard-Smithsonian CfA, 60 Garden St MS 10, Cambridge MA 02138 USA
}

\begin{abstract}
We present new observations of the emission spectrum of the hot Jupiter TrES-4 designed to test the theory that the presence of temperature inversions in the atmospheres of these planets are correlated with the amount of radiation received by the planet. Our observations reveal that TrES-4 has an emission spectrum similar to that of HD 209458b, which requires the presence of an inversion layer high in the atmosphere and water emission bands in order to explain the observed features, providing additional support for that theory. We also present new observations of the thermal phase curve of HD 189733b at $24 \mu \mathrm{m}$, which we combine with our previous observations at $8 \mu \mathrm{m}$ to examine how circulation in this planet's atmosphere varies as a function of depth. We discuss the relationship between the strength of the day-night circulation on both planets and their other observable properties, in particular their emission spectra.
\end{abstract}

\section{Introduction}

Transiting extrasolar planets offer a unique opportunity to study the diversity of planetary atmospheres, and also provide an important testing ground for models of these atmospheres. The "hot Jupiters" are a prime example: although these planets have masses similar to the gas giant planets of the Solar system, they orbit at less than $0.05 \mathrm{AU}$ from their parent stars. Because the time scale for tidal synchronization is short compared to the ages of these systems, these planets are expected to be tidally locked, with permanent day and night sides. This presents a significant challenge for planetary atmosphere models, as the equilibrium temperatures are substantially higher $(1000-2000 \mathrm{~K})$ and the atmospheric circulation patterns significantly different than those of Jupiter. The hottest of these planets have temperatures comparable to those of the coolest stars, placing them in a unique parameter space with potentially exotic atmospheric chemistry. As a result of this intense and highly asymmetric irradiation and their presumably slower rotation rates, the atmospheric dynamics of these planets are also expected to differ significantly from those of the gas giant planets in the solar system.

In the following sections we present new examples of two distinct types of observations designed to characterize the properties of these unique atmospheres. In $\S 2$ we examine secondary eclipse observations of the hot Jupiter TrES-4, the most highly irradiated planet to be observed with Spitzer to date. In $\S 3$ we show new $24 \mu \mathrm{m}$ observations of the phase curve of HD $189733 \mathrm{~b}$, and compare them to $8 \mu \mathrm{m}$ phase curve for this planet from Knutson et al. (2007a). Both observations highlight the multiple connections between atmospheric circulation and the shape of the day-side emission spectrum on these unusual gas giant planets.

\section{Secondary Eclipse Measurements for TrES-4}

By measuring the decrease in flux as these planets move behind their parent stars, it is possible to characterize the light emitted by the day side of these planets, and to construct a rough spectrum (Charbonneau et al. 2005, Deming et al. 2005, 2006, Grillmair 


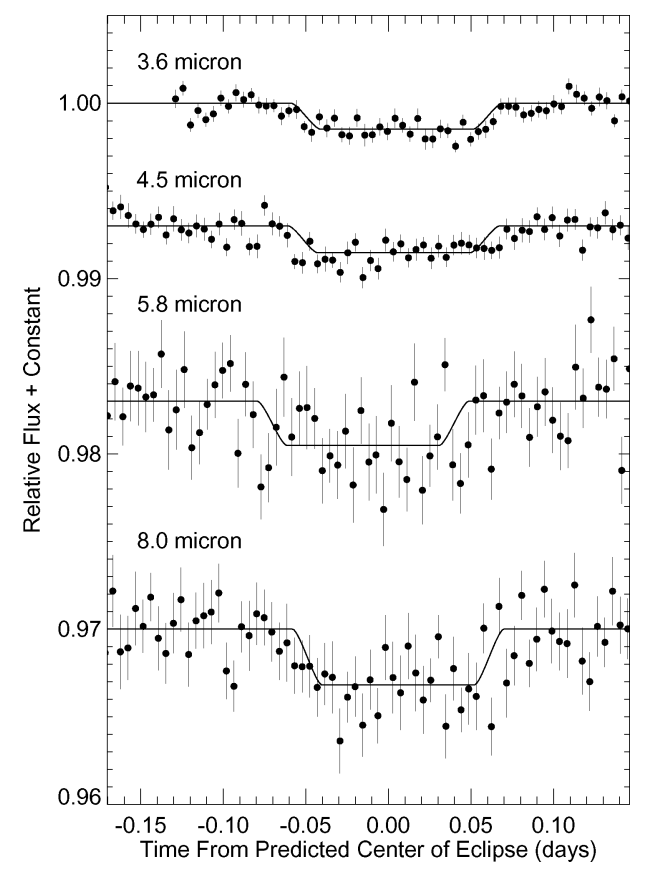

Figure 1. Secondary eclipse of TrES-4 observed on UT 2007 Oct. 19 at 3.6 and $5.8 \mu \mathrm{m}$ and on UT 2007 Oct. 22 at 4.5 and $8.0 \mu \mathrm{m}$, with best-fit eclipse curves overplotted. Data has been normalized to remove detector effects and binned in 6.6 minute intervals.

et al. 2007, Richardson et al. 2007, Harrington et al. 2007, Deming et al. 2007, Demory et al. 2007, Charbonneau et al. 2008, Knutson et al. 2008a, Machalek et al. 2008). These observations indicate that there are two distinct classes of hot Jupiter atmospheres. One class of planets, including HD 189733b (Deming et al. 2006, Grillmair et al. 2007, Charbonneau et al. 2008) and TrES-1 (Charbonneau et al. 2005), have emission spectra that are consistent with standard 1D cloud-free atmosphere models for these planets (Hubeny et al. 2003, Sudarsky et al. 2003, Seager et al. 2005, Barman 2005, 2008, Fortney et al. 2005, 2006a,b, Burrows et al. 2005, 2006, 2008). These model spectra are dominated by strong absorption features from $\mathrm{CO}$ and $\mathrm{H}_{2} \mathrm{O}$ in the infrared. In contrast, the emission spectra of planets such as HD 209458b (Deming et al. 2005, Richardson et al. 2007, Burrows et al. 2007, Knutson et al. 2008a) and XO-1b (Machalek et al. 2008) require models with a temperature inversion between $0.1-0.01$ bars and water bands in emission instead of absorption (Hubeny et al. 2003, Fortney et al. 2006a, Burrows et al. 2007, 2008, Fortney et al. 2008) in order to explain the observed features.

Although the nature of the high-altitude absorber needed to produce temperature inversions in the atmospheres of HD 209458b and XO-1b is currently unknown, there appears to be an intriguing connection between the equilibrium temperature of the planet in question and the presence or absence of an inversion. HD 209458b, which has an inversion, receives approximately twice as much radiation from its primary as either HD 189733 b or TrES-1, which do not have inversions. XO-1b is an exception to this rule, as it apparently possesses a temperature inversion despite levels of irradiation comparable to those of HD 189733b and TrES-1. However XO-1b orbits a metal-rich star and thus may have a fundamentally different atmospheric composition than that of either HD 189733 b or HD 209458 b, which orbit stars with solar-type metallicities. Leaving XO-1b 


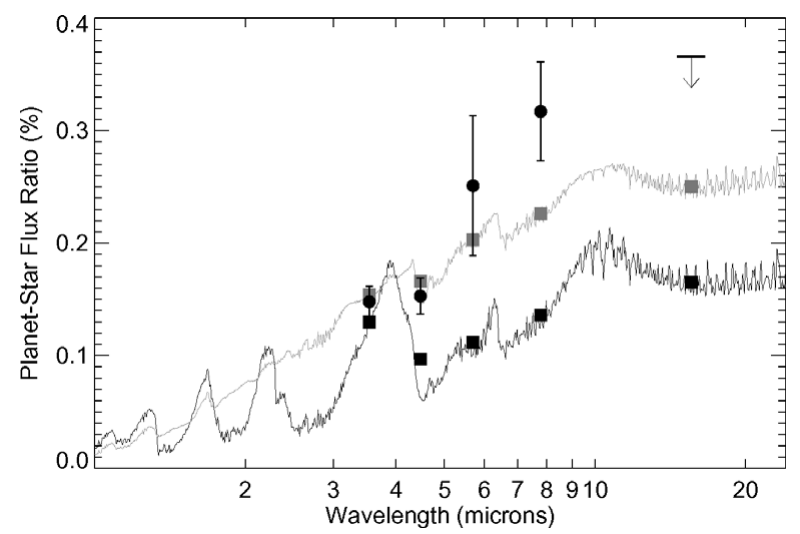

Figure 2. Day-side planet-star flux ratios for TrES-4 as determined from measurements of the secondary eclipse depth in the four IRAC bandpasses (black circles). The horizontal bar and arrow at $16 \mu \mathrm{m}$ show the $2 \sigma$ upper limit on the eclipse depth in this bandpass. The black line corresponds to the default model (no temperature inversion) with a redistribution parameter $P_{n}=0.1$, which describes the case where $10 \%$ of the incident energy is redistributed to the night side. The grey line correspond to a model with $P_{n}=0.1$ and an additional optical absorber at high altitudes, which produces a thermal inversion around pressures of 0.001 bar (Burrows et al. 2007, 2008). Squares show the values for these models after integrating over the Spitzer bandpasses.

aside for the moment, it is possible that the increased irradiation experienced by HD $209458 \mathrm{~b}$ relative to these other planets might have crossed a threshold beyond which non-equilibrium compounds form through photolysis (Burrows et al. 2008), and that these, in turn, provide the additional opacity at altitude needed to produce the inversion. Similarly, it has been suggested (Hubeny et al. 2003, Burrows et al. 2007, 2008, Fortney et al. 2008) that the presence of gas-phase $\mathrm{TiO}$ or $\mathrm{VO}$ in the atmosphere might also provide the necessary opacity. These compounds are predicted to have condensed out of the relatively cool atmospheres of HD $189733 \mathrm{~b}$ and TrES-1, whereas they might still remain in gas phase in the hottest regions on the day side of HD 209458b.

TrES-4 (Mandushev et al. 2007) orbits a $1.22 \mathrm{M}_{\odot}$ star with a period of only 3.55 days, and as a result it receives twice as much radiation as HD 209458b, and four times as much as HD 189733b, TrES-1, and XO-1. If planets with higher levels of irradiation are more likely to have temperature inversions, then TrES-4 is an ideal candidate. We tested this theory by observing three secondary eclipses of TrES-4 over a period of two weeks in Oct. 2007 using the Spitzer Space Telescope, obtaining data at 3.6, 4.5, 5.8, 8.0, and 16 $\mu \mathrm{m}$. We used the Infrared Spectrograph (IRS) on UT 2007 Oct. 8 to observe an eclipse in the $16 \mu \mathrm{m}$ peak-up imaging mode. Next we observed a secondary eclipse on UT 2007 Oct. 19 using the Infrared Array Camera (IRAC), obtaining data simultaneously at 3.6 and $5.8 \mu \mathrm{m}$, and last we observed a secondary eclipse on UT 2007 Oct. 22 at 4.5 and $8.0 \mu \mathrm{m}$. We constructed a time series in each of the four IRAC and IRS channels using the methods described in Knutson et al. (2008a) and Charbonneau et al. (2008), and we present a detailed description of our analysis of this data in Knutson et al. (2008c).

Fig. 1 shows the time series for the four IRAC bandpasses. We do not detect the eclipse in the $16 \mu \mathrm{m}$ IRS bandpass, and instead place a $2 \sigma$ upper limit on the depth of the secondary eclipse in this bandpass. We then compare the secondary eclipse depths in the four IRAC bandpasses to the predictions from atmosphere models for this planet (see Fig. 2) from Knutson et al. (2008c). These models employ the same formalism described 
in Burrows et al. $(2007,2008)$, which uses a dimensionless redistribution parameter $\mathrm{P}_{n}$ that, in approximate fashion, accounts for the cooling of the dayside and the warming of the nightside by zonal winds near an optical depth of order unity. The larger $\mathrm{P}_{n}$ is, the cooler is the dayside atmosphere and the lower the emergent planetary flux at superior conjunction. $\kappa_{\text {extra }}$ is the absorptive opacity in the optical at altitude (here in $\mathrm{cm}^{2} / \mathrm{g}$ ) used to create a temperature inversion.

Fig. 2 shows two models with varying values for $\kappa_{\text {extra }}$. The standard non-inverted model (black line in Fig. 2, $\kappa_{\text {extra }}=0 \mathrm{~cm}^{2} / \mathrm{g}$ ) is clearly inconsistent with the observed fluxes from TrES-4 at wavelengths longer than $4 \mu \mathrm{m}$, whereas the model with extra opacity (grey line in Fig. 2, $\kappa_{\text {extra }}=0.1 \mathrm{~cm}^{2} / \mathrm{g}$ ) and a thermal inversion provides a much better fit to these long-wavelength data. We note that both models employ a relatively small value for $P_{n}$, corresponding to minimal circulation between the day- and nightside hemispheres. Decreasing the amount of day-night circulation in the TrES-4 models increases the effective temperature of the day-side atmosphere, which in this case provides a better match to the relatively high fluxes observed during secondary eclipse.

\section{3. $24 \mu \mathrm{m}$ Phase Curve for HD 189733b}

By observing the changes in the planet's thermal emission as a function of orbital phase, we can directly determine the day-night temperature difference for these hot Jupiters. Harrington et al. (2006) reported the first detection of these phase variations for the nontransiting planet $v$ Andromedae b at $24 \mu \mathrm{m}$. If one makes reasonable assumptions about the predicted size and temperature of $v$ Andromedae $\mathrm{b}$ based on its mass and distance from its star, the large size of the observed phase variation implies a large day-night temperature difference and correspondingly inefficient thermal homogenization between the day and night sides. Cowan et al. (2007) made similar $8 \mu \mathrm{m}$ observations of three other systems, HD 209458, HD 179949, and 51 Peg, of which only HD 209458 is a transiting system. They report a detection for the non-transiting system HD 179949, implying a large day-night temperature difference similar to that of $v$ Andromedae b.

The best-constrained determination of a phase curve for a hot Jupiter comes from Knutson et al. (2007), hereafter known as Paper I, where we present $8 \mu \mathrm{m}$ observations of the transiting planet HD 189733b spanning slightly more than half of its orbit. The high cadence of these data made it possible for us to fit the resulting phase curve with an "orange-slice" model for the planet (Cowan et al. 2008) consisting of twelve longitudinal strips of constant brightness. The small size of the observed phase variation argued for highly efficient thermal homogenization between the two hemispheres, in contrast to the large day-night temperature differences inferred for $v$ Andromedae b and HD 179949b.

Although this longitudinal temperature map provides a wealth of information about the circulation within HD 189733b's atmosphere, its interpretation is complicated by the fact that the altitude of the atmospheric layer corresponding to the derived map depends on the atmospheric opacity at $8 \mu \mathrm{m}$. For wavelengths where the opacity is low the effective photosphere of the planet is located deep in the atmosphere, where the pressures and temperatures are correspondingly higher. In their dynamical models Cooper \& Showman (2005), Dobbs-Dixon \& Lin (2008), and Showman et al. (2008) show that temperatures are likely to become increasingly homogenized at these higher pressures, as the radiative time scale increases relative to the advective time scale. Thus we would expect that observations of the same planet at different wavelengths might show varying brightness contrasts between the day and night sides, depending on how deep into the atmosphere we are looking at each wavelength. This also means that there is an inherent difficulty 
in comparing observations of one planet ( $v$ Andromedae b) at $24 \mu \mathrm{m}$ with observations of other planets (HD 179949b and HD 189733b) at $8 \mu \mathrm{m}$.

In Knutson et al. (2008b), hereafter Paper II, we present new observations of the phase variation of HD 189733b at $24 \mu \mathrm{m}$, spanning the same part of the planet's orbit as the previous observations at $8 \mu \mathrm{m}$ described in Paper I. We describe our analysis of these images in Paper II. We find that the planet's atmosphere exhibits only a modest variation in the day/night brightness temperatures at both 8 and $24 \mu \mathrm{m}$ when compared to radiativeequilibrium predictions for highly-irradiated, tidally-locked planets. This implies efficient transport of thermal energy from the day side to the night side by atmospheric winds at the level of both the 8 and $24 \mu \mathrm{m}$ photospheres. The planet's 8 and $24 \mu \mathrm{m}$ phase curves both reach a peak before the secondary eclipse, indicating that the hottest region on the day side is shifted 30-40 degrees east of the substellar point at the location of both photospheres and providing additional evidence for the horizontal and/or vertical advection of the temperature field in the planet's atmosphere. The similarities between the phase curves at 8 and $24 \mu \mathrm{m}$ suggests that either both wavelengths sense similar atmospheric pressures or that the circulation behaves in a fundamentally similar fashion across the relatively modest (factor of $2-3$ ) range in pressures that are sensed here according to atmosphere models. The uncertain abundance of methane in the planet's atmosphere complicates this picture (Swain et al. 2008), but based on other data we can definitively

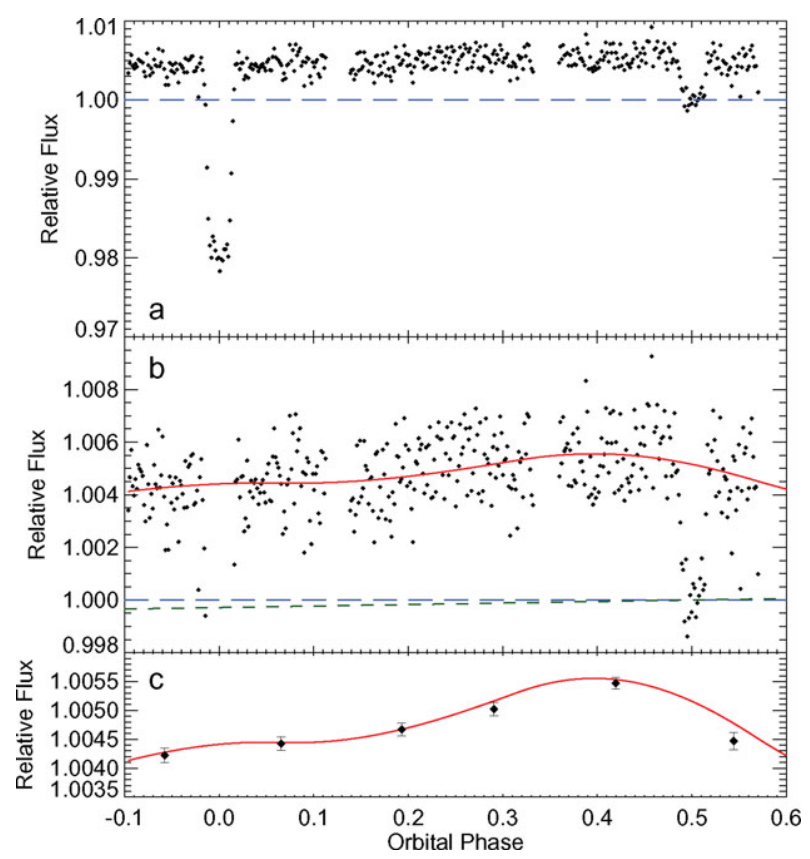

Figure 3. Phase variation observed for HD 189733b by Spitzer in the MIPS $24 \mu \mathrm{m}$ bandpass, with transit and secondary eclipse visible. The data are binned in 6.3-minute intervals. The stellar flux as measured at the center of the secondary eclipse has been normalized to unity (long-dashed line). Panels $\mathbf{a}, \mathbf{b}$ and $\mathbf{c}$ show the same data, but in $\mathbf{b}$ and $\mathbf{c}$ the $\mathbf{y}$ axis is expanded to show the scale of the observed variation. The bin size is also increased significantly in $\mathbf{c}$ to allow for a better comparison with the model fit. The solid line in $\mathbf{b}$ and $\mathbf{c}$ is the phase curve for the best-fit four slice model, and the short-dashed line in $\mathbf{b}$ shows the expected change in the star's flux as a result of the rotational modulation in the visibility of star spots over the period of the observations. 
rule out the presence of an opaque cloud layer above the range of the 8 and $24 \mu \mathrm{m}$ contribution functions that, if present, might affect these observations (Charbonneau et al. 2008, Barman 2008).

Our observations at $24 \mu \mathrm{m}$ confirm that HD 189733b's phase curve is fundamentally different than that of $v$ Andromedae b or HD 179949b. Although these three planets constitute a very limited sample, this result would seem to point towards the existence of two distinct classes of hot Jupiter atmospheres, characterized by either efficient or inefficient thermal homogenization between the day and night sides of the planet. This is particularly interesting in light of the results discussed in $\S 2$, which indicate that the day-side emission spectra of these planets can also be divided into two classes depending on whether or not the planet has a thermal inversion. It is possible that the presence of a temperature inversion and the degree of thermal homogenization are connected. Fortney et al. (2008) note that planets with temperature inversions absorb more of the incident flux higher in their atmospheres, where the radiative time scale is short compared to the advective time scale. They argue that this would naturally lead to large day-night temperature differences for these planets, while planets with lower levels of irradiation and no temperature inversions would be more homogenized. Both $v$ Andromedae $\mathrm{b}$ and HD 179949b have higher levels of incident flux than HD 189733b and TrES-1, placing them in the same class as HD 209458b and TrES-4, which both have inversions. Unfortunately neither $v$ Andromedae b nor HD $179949 \mathrm{~b}$ are eclipsing, making it difficult to check directly for the presence of a temperature inversion. A better test would be to measure the day-night temperature difference for HD 209458b, which clearly does have a temperature inversion, or HD 149026b, whose high $8 \mu \mathrm{m}$ brightness temperature (Harrington et al. 2007) strongly favors the presence of a temperature inversion (Fortney et al. 2006a, Burrows et al. 2008). We have obtained such observations of both planets, and will report on the results in a future paper.

\section{Conclusions: Future Directions}

In the sections above we describe a picture in which the presence of thermal inversions is connected to the degree of irradiation experienced by the planet, as well as a parallel suggestion that planets with inversions may have weaker day-night circulation and correspondingly large day-night temperature differences. Our observations of TrES-4 and HD 189733b appear to support this theory: the day-side emission spectrum of the highlyirradiated TrES-4 is best fit by a model with a temperature inversion and weak day-night circulation, while our 8 and $24 \mu \mathrm{m}$ observations of the phase curve of HD 189733b indicate that this planet has a small day-night temperature difference, and Charbonneau et al. (2008) find that the planet is best fit by a model without a temperature inversion.

Although our observations of TrES-4 and HD 189733b are in good agreement with this picture, they do not distinguish between competing theories for the nature of the optical absorber responsible for the creation of the inversions. Furthermore, new observations of XO-1 presented by Machalek et al. (2008) appear to conflict with this relatively simple picture. If we are to fully understand the origins of these inversions and their effects on the day-night circulation on these planets, a much larger sample will be required than the seven systems with published Spitzer observations. Although Spitzer will deplete its supply of cryogen in spring 2009, the 3.6 and $4.5 \mu \mathrm{m}$ channels will continue to function at full sensitivity, and a two-year mission using these two channels was recently approved by NASA. Observations of the secondary eclipse in these two channels should be sufficient to distinguish between planets with and without temperature inversions, as demonstrated by our discussions above, and it may also be possible to obtain phase curves in these 
two channels once the problem of intra-pixel sensitivity has been dealt with adequately. Observations of hot Jupiters spanning a range of stellar metallicities, levels of irradiation, surface gravities, and orbital periods might reveal important connections between these properties, the presence of a temperature inversion, and the strength of the day-night circulation.

\section{Acknowledgements}

This work is based on observations made with the Spitzer Space Telescope, which is operated by the Jet Propulsion Laboratory, California Institute of Technology, under contract to NASA. Support for this work was provided by NASA through an award issued by JPL/Caltech. HAK was supported by a National Science Foundation Graduate Research Fellowship.

\section{References}

Barman, T. S., Hauschildt, P. H., \& Allard, F. 2005, ApJ, 632, 1132

Barman, T. S. 2008, ApJ, 676, L61

Burrows, A., Hubeny, I., \& Sudarsky, D. 2005, ApJ, 625, L135

Burrows, A., Sudarsky, D. \& Hubeny, I. 2006, ApJ, 650, 1140

Burrows, A., Hubeny, I., Budaj, J., Knutson, H. A., \& Charbonneau, D. 2007, ApJ, 668, L171

Burrows, A., Budaj, J., \& Hubeny, I. 2008, ApJ, in press, astro-ph/0709.4080

Charbonneau, D., et al. 2005, ApJ, 626, 523

Charbonneau, D., et al. 2008, ApJ, submitted, astro-ph/0802.0845

Cooper, C. S. \& Showman, A. P. 2005, ApJ, 629, L45

Cowan, N. B., Agol, E., \& Charbonneau, D. 2007, MNRAS, 379, 641

Cowan, N. B. \& Agol, E. 2008, ApJ, 678, L129

Deming, D., Seager, S., \& Richardson, L. J. 2005, Nature, 434, 740

Deming, D., Harrington, J., Seager, S., \& Richardson, L. J. 2006, ApJ, 644, 560

Deming, D. et al. 2007, ApJ, 667, L199

Demory, B.-O. et al. 2007, A\&A A, 475, 1125

Dobbs-Dixon, I. \& Lin, D. N. C. 2008, ApJ, 673, 513

Fortney, J. J., Marley, M. S., Lodders, K., Saumon, D., \& Freedman, R. 2005, ApJ, 627, L69

Fortney, J. J., et al. 2006a, ApJ, 652, 746

Fortney, J. J., Saumon, D., Marley, M. S., Lodders, K., \& Freedman, R. S. 2006b, ApJ, 642, 495

Fortney, J. J., Lodders, K., Marley, M. S., \& Freedman, R. S. 2008, ApJ, 678, 1419

Grillmair, C. J. et al. 2007, ApJ, 658, L115

Harrington, J. et al. 2006, Science, 314, 623

Harrington, J., Luszcz, S., Seager, S., Deming, D., \& Richardson, L. J. 2007, Nature, 447, 691

Hubeny, I., Burrows, A., \& Sudarsky, D. 2003, ApJ, 594, 1011

Knutson, H. A., et al. 2007, Nature, 447, 183

Knutson, H. A., et al. 2008a, ApJ, 673, 526

Knutson, H. A., et al. 2008b, ApJ, submitted, astro-ph/0802.1705

Knutson, H. A., Charbonneau, D., Burrows, A., O'Donovan, F. T., \& Mandushev, G. 2008c, ApJ, submitted

Machalek, P., et al. 2008, ApJ in press, astro-ph/0805.2418

Richardson, L. J., Deming, D., Horning, K., Seager, S., \& Harrington, J. 2007, Nature, 445, 892

Seager, S. et al. 2005, ApJ, 632, 1122

Showman, A. P. et al. 2008, ApJ in press, astro-ph/0802.0327

Sudarsky, D., Burrows, A., \& Hubeny, I. 2003, ApJ, 588, 1121

Swain, M. R., Vasisht, G., \& Tinetti, G. 2008, Nature, 452, 329 